\title{
Der Bundestag in der Europäischen Union - (Ein)Blick von innen
}

\author{
Christiane Barnickel
}

Bisher wurde die Leistung nationaler Parlamente im europäischen Politikzyklus lediglich „von außen“ aus der Perspektive eines spezifischen (impliziten oder expliziten) Legitimitätsdenkens bewertet und damit zusammenhängend auch normativ vor dem Hintergrund der jeweils favorisierten Position des Bundestags. Der „Blick von innen“, also ob, unter welchen Bedingungen und mit welchen (konkurrierenden) Begründungen Bundestagsabgeordnete europäisches Regieren als legitim erachten, fehlte bisher. ${ }^{1}$

Die im parlamentarischen Raum artikulierten Begründungsmuster für die Legitimität oder Illegitimität europäischen Regierens differenzieren sich zunehmend aus, wie die nachstehenden Zitate veranschaulichen: „Mehr Demokratie schaffen wir durch eine Stärkung der Parlamente [...]" (Thomas Silberhorn 2008a, 15854A)² - so die Auffassung eines MdB, wie sie in einer Debatte zum Vertrag von Lissabon geäußert wurde. Fast diametral entgegengesetzt mutet die Diagnose an, dass „die auf europäischer Ebene getroffenen Entscheidungen [...] nicht mehr hinreichend [...] legitimiert werden [können]“ (Peter Gauweiler 2008b, 16610D). Mit dem lange beschworenen „permissiven Konsens“3 in der Europapolitik haben diese Aussagen nicht mehr allzu viel zu tun und es ist unklar, welchem Legitimitätsdenken die konträren Äußerungen entspringen.

In der wissenschaftlichen Debatte lässt sich Ähnliches an der fortdauernden Auseinandersetzung um das Demokratie- und/oder Legitimitätsdefizit der Europäischen Union nachzeichnen. Hier zeigt sich, dass bei der Überprüfung, ob europäisches Regieren hinreichend legitimiert ist, auf unterschiedliche Begründungsmodi für legitime Herrschaft rekurriert wird. ${ }^{4}$ Abgehoben wird sowohl auf institutionelle als auch auf strukturelle Faktoren, die vor

1 Ausnahmen, allerdings mit anderer Stoßrichtung, bilden beispielsweise Katrin Auel, The Europeanisation of the German Bundestag: Institutional Change and Informal Adaptation, in: German Politics, 15. Jg. (2006), H. 3, S. 249 - 268; dies. / Arthur Benz, The Politics of Adaptation: The Europeanisation of National Parliamentary Systems, in: The Journal of Legislative Studies, 11. Jg. (2005), H. 3, S. 372 - 393; Bernhard Wessels, Roles and Orientations of Members of Parliament in the EU Context: Congruence or Difference? Europeanisation or not?, in: The Journal of Legislative Studies, 11. Jg. (2005), H. 3/4, S. $446-465$.

2 Zur besseren Lesbarkeit werden in Referenzen auf Plenarprotokolle Sprecher, Jahr und Minuskel angegeben. Es wurde auf folgende Protokolle zurückgegriffen: Deutscher Bundestag, Plenarprotokoll 16/151 vom 13. März 2008. Stenografischer Bericht, Berlin 2008a; ders., Plenarprotokoll 16/157 vom 24. April 2008. Stenografischer Bericht, Berlin 2008b; ders., Plenarprotokoll 16/229 vom 1. Juli 2009. Stenografischer Bericht, Berlin 2009a; ders., Plenarprotokoll 16/232 vom 26. August 2009. Stenografischer Bericht, Berlin 2009b; ders., Plenarprotokoll 16/233 vom 8. September 2009. Stenografischer Bericht, Berlin 2009c.

3 Leon Lindberg/ Stuart Scheingold, Europe's Would-Be Polity: Patterns of Change in the European Community, Englewood Cliffs 1970, S. 41.

4 Vgl. die konträren Positionen bei Giandomenico Majone, Europe's 'Democratic Deficit': The Question of Standards, in: European Law Journal, 4. Jg. (1998), H. 1, S. 5 - 38; Andrew Moravcsik, In Defense of the 'Democratic Deficit': Reassessing Legitimacy in the European Union, in: Journal of Common Market Studies, 40. Jg. (2002), H. 4, S. 603 - 624; dem gegenüber Andreas Føllesdal I Simon Hix, Why There is a Democratic Deficit in the EU: A Response to Majone and Moravcsik, in: Journal of Common Market Studies, 44. Jg. (2006), H. 3, S. 533 - 562. 
der Folie einer (impliziten oder expliziten) Idealvorstellung durchdekliniert werden. ${ }^{5}$ Daneben finden sich Analysen sowohl der Eliten- als auch der Bevölkerungseinstellungen, die der Akzeptanz europäischen Regierens nachgehen. Allerdings fragen diese zumeist nicht nach tieferliegenden Erklärungsfaktoren für (divergierende) Einstellungen und vernachlässigen somit, auf welchen Grundfundamenten des Legitimitätsdenkens diese Einstellungen aufbauen. ${ }^{6}$

Gerade im Hinblick auf Parlamente stellt diese Wissenslücke einen problematischen Befund dar, denn sie sind nicht nur das Verbindungsglied zwischen Bevölkerung und politischem System, sondern wurden im Laufe der Entwicklung der europäischen Verträge zunehmend mit einer legitimatorischen Funktion für europäisches Regieren beauftragt. Demgegenüber steht ihre diagnostizierte Schwäche als „Verlierer“7 der Integration. Es handelt sich hier um eine doppelte Kontroverse: Einerseits geht es um die normative Bewertung der Europäisierung nationaler Parlamente, andererseits um ihre adäquate Einbindung in den europäischen Politikprozess. ${ }^{8}$ Beide Aspekte sind eng verwoben mit Legitimitätsvorstellungen und der impliziten Annahme, Unterschiede hierin gingen Hand in Hand mit unterschiedlichen Präferenzen für Rollen und Funktionen von Parlamenten. ${ }^{9}$

Daher sollen im Folgenden, erstens, potentiell unterschiedliche Begründungsmuster von Bundestagsabgeordneten für (il)legitimes europäisches Regieren ergründet und, zweitens, ermittelt werden, inwiefern diese mit unterschiedlichen Präferenzen für auszuübende Parlamentsfunktionen verbunden sind.

5 Vgl. Peter Graf Kielmansegg, Integration und Demokratie, in: Markus Jachtenfuchs / Beate KohlerKoch (Hrsg.), Europäische Integration, Opladen 2003, S. 49 - 83; Fritz W. Scharpf, Regieren in Europa: effektiv und demokratisch, Frankfurt am Main / New York 1999. Für systematisierende Bestandsaufnahmen der Debatte vgl. Erik Oddvar Eriksen / John Erik Fossum, Europe in Search of Legitimacy: Strategies of Legitimation Assessed, in: International Political Science Review, 25. Jg. (2004), H. 4, S. 435 - 459; Andreas Føllesdal, Survey Article: The Legitimacy Deficits of the European Union, in: The Journal of Political Philosophy, 14. Jg. (2006), H. 4, S. $441-468$; Christopher Lord / Paul Magnette, E Pluribus Unum? Creative Disagreement about Legitimacy in the EU, in: Journal of Common Market Studies, 42. Jg. (2004), H. 1, S. 183 - 202. Für eine Übersicht zur Debatte vgl. Holger Huget, Demokratisierung der EU, Wiesbaden 2007.

6 Vgl. Liesbet Hooghe, Europe Divided? Elites vs. Public Opinion on European Integration, in: European Union Politics, 4. Jg. (2003), H. 3, S. 281 - 304; Markus Jachtenfuchs, Ideen und Integration. Verfassungsideen in Deutschland, Frankreich und Großbritannien und die Entwicklung der EU, Mannheim 1999, http://www.hertie-school.org/binaries/addon/321_habil.pdf (Abruf am 13. Mai 2011).

7 Andreas Maurer / Wolfgang Wessels (Hrsg.), National Parliaments on their Ways to Europe. Losers or Latecomers?, Baden-Baden 2001.

8 Vgl. Klaus von Beyme, Niedergang der Parlamente, in: Internationale Politik, 53. Jg. (1998), H. 4, S. 21 - 30, S. 27; Simon Hix / Klaus H. Goetz, Introduction: European Integration and National Political Systems, in: West European Politics, 23. Jg. (2000), H. 4, S. 1 - 26, S. 6; Annette Elisabeth Töller, Dimensionen der Europäisierung - Das Beispiel des Deutschen Bundestages, in: ZParl, 35. Jg. (2004), H. 1, S. 25 - 50, S. 30 ff.; mit anderer Stoßrichtung Tapio Raunio / Simon Hix, Backbenchers Learn to Fight Back: European Integration and Parliamentary Government, in: West European Politics, 23. Jg. (2000), H. 4, S. 142 - 168, S. 159.

9 Vgl. Andreas Follesdal, a.a.O. (Fn. 5), S. 445; Wolfgang Ismayr, Der Deutsche Bundestag im politischen System der Bundesrepublik Deutschland, Opladen 2001, S. 34, S. 41; Bernhard Wessels 1 Richard S. Katz, Introduction: European Parliament, National Parliaments and European Integration, in: dies. (Hrsg.), The European Parliament, National Parliaments and European Integration, Oxford 1999, S. 1 - 18, S. 3. 


\section{Legitimitätsmuster und Bundestagsfunktionen - Perspektiven der MdBs}

Für die Analyse der Legitimitätsvorstellungen und des Funktionsverständnisses wurden Debattenbeiträge des Bundestages herangezogen und in einem explorativen Prozess des Verstehens (latente) Denkmuster der MdBs herausgefiltert. Aus zwei Gründen legen die Plenardebatten zum Vertrag von Lissabon und der nationalen Begleitgesetzgebung eine Aktivierung von Deutungsmustern nahe. Eine Aktivierung ist wahrscheinlich zu Zeiten struktureller Veränderungen, die zudem als problematisch angesehen werden. ${ }^{10} \mathrm{Im}$ vorliegenden Fall bildet die Vertragsrevision die bestehende Ordnung um. Bereits die Klagen vor dem Bundesverfassungsgericht belegen, dass die Normveränderung kontrovers ist. ${ }^{11}$ Folglich wurden die Debatten der Plenarsitzungen vom 13. März 2008, 24. April 2008, 1. Juli 2009, 26. August 2009 und 8. September 2009 ausgewählt. ${ }^{12}$ Eingang in die Analyse fanden die Beiträge aller MdBs, die sich mit einer Rede, Kurzintervention, Zwischenfrage und/oder einer zu Protokoll gegebenen Erklärung in die Debatten einbrachten. Insgesamt sind dies Äußerungen von 25 MdBs. Regierungsvertreter, die in den Debatten zu Wort kamen, wurden nicht einbezogen, da das Forschungsinteresse auf die spezifischen parlamentarischen Positionen gerichtet war. Auch blieben die Reden jener MdBs unberücksichtigt, deren Beiträge keine Hinweise auf Legitimitätsvorstellungen oder die Positionierung des Bundestags enthielten, da daraus nicht auf den der Fragestellung zu Grunde liegenden Zusammenhang geschlossen werden konnte. ${ }^{13}$

Das Codierschema ist an die von Andreas Wimmel ${ }^{14}$ entwickelte Heuristik der in der Literatur verhandelten Kategorien zur Legitimitätsbewertung der EU angelehnt. Folgende von ihm identifizierte Dimensionen dienten der Orientierung für die empirische Analyse: Legitimationskonzepte (Legalität, Zustimmung/empirische Legitimität, normative Rechtfertigung), das Legitimationsobjekt „Politische Ordnung der EU“, auf das sich die Konzep-

10 Vgl. Ulrich Oevermann, Zur Analyse der Struktur von Sozialen Deutungsmustern, in: Sozialer Sinn, 2. Jg. (2001), H. 1, S. 3 - 33, S. 18.

11 Alle analysierten Debatten fallen in die 16. Wahlperiode, Regierungszeit der Großen Koalition. Zum Vertrag von Lissabon und nationaler Begleitgesetzgebung vgl. neben Vertrags- und Gesetzestext zum Beispiel Daniela Kietz, Die Nationalen Parlamente und die Beteiligungsrechte des Bundestags, in: Julia Lieb / Nicolai von Ondarza / Torsten Schütz (Hrsg.), Der Vertrag von Lissabon und seine Umsetzung. SWP-Webdossier, Berlin 2010, S. 56 - 67; zu Klagen und Urteil Franz C. Mayer, Dichtung und Wahrheit im europäischen Verfassungsdiskurs - Anmerkungen zum Beitrag von K.A. Schachtschneider, in: Leviathan, 36. Jg. (2008), H. 4, S. 477 - 489; Karl Albrecht Schachtschneider, Verfassungsrechtliche Argumente gegen den Vertrag von Lissabon, in: Leviathan, 36. Jg. (2008), H. 3, S. 317 - 343; Bundesverfassungsgericht, 2 BvE 2/08 vom 30. Juni 2009; Martin Höpner / Stephan Leibfried / Marcus Höreth / Fritz W. Scharpf / Michael Zürn, Kampf um Souveränität? Eine Kontroverse zur europäischen Integration nach dem Lissabon-Urteil des Bundesverfassungsgerichts, in: PVS, 51. Jg. (2010), H. 2, S. 323 - 355; zum Abstimmungsverhalten der MdBs vgl. Deutscher Bundestag, a.a.O. (Fn. 2), 2008b, S. 16476 ff., S. 16482 ff.; ders., a.a.O. (Fn. 2), 2009 c, S. 26359 f.

12 Bei den Debatten vom 13. März 2008 und 24. April 2008 handelt es sich um die Beratungen zum Entwurf des Gesetzes zum Vertrag von Lissabon, sowie zu den Begleitgesetzen zur Änderung des GG und zur Stärkung der Rechte der Parlamente. Am 1. Juli 2009 fand eine Debatte zum Urteil des BVerfG statt. Die beiden verbleibenden Debatten sind die Beratungen zur Revision der Begleitgesetzgebung nach dem Verfassungsgerichtsurteil.

13 Gleichwohl wurden die Reden Letzterer codiert, um nicht Gefahr zu laufen, Beiträge vorschnell aus der Analyse zu nehmen.

14 Vgl. Andreas Wimmel, Die demokratische Legitimität europäischen Regierens: ein Labyrinth ohne Ausgang?, in: Integration, 31. Jg. (2008), H. 1, S. 48 - 64. 
te beziehen können sowie die Legitimationsvariablen Input, Throughput und Output. ${ }^{15}$ Die verbleibenden Objektdimensionen (Institutionen und Politikentscheidungen) wurden einbezogen, sofern sie Auswirkungen auf die Legitimität des Gesamtsystems zeitigen. Ähnlich wurde mit dem Objekt „Nationale politische Ordnung“ verfahren, das eingefügt wurde, um der Konnektivität der Ebenen Rechnung tragen zu können. ${ }^{16}$ Weitere Codes, insbesondere Untercodes, wurden - in kontinuierlichem Abgleich mit einschlägiger Theorie - weitgehend induktiv generiert. ${ }^{17}$ Am Ende lagen 1.682 codierte Textstellen vor. Aus Vergleich und Kontrastierung wurden die Standpunkte zu vier Mustern gruppiert und zur Funktionsbestimmung des Bundestags in Bezug gesetzt.

\subsection{Souveränitätsreflex zur Wahrung der nationalen Demokratie}

Ein erstes Legitimitätsmuster lässt sich als „Wahrung der nationalen Demokratie“ umreißen und korrespondiert mit einer kontrollierend-kompetenzwahrenden Positionierung des Bundestags. ${ }^{18}$ Input- und Throughput stehen im Fokus, der Output ist kaum legitimationsrelevant. In Anbetracht der Stärke des Inputs und dessen Verbindung zum Throughput wird legitimes mit demokratischem Regieren, verstanden als Wahl und Transformation des Bürgerwillens, zuvorderst durch Parlamente, gleichgesetzt. Für Peter Gawweiler und Henry Nitzsche ist demokratisches Regieren in Ermangelung eines demos auf EU-Ebene unabhängig von der mitgliedstaatlichen Legitimation nicht möglich. Mithin kann „europäische Politik [... nicht als Herrschaft durch das Volk legitimiert werden "19. Das Europäische Parlament (EP) und dessen Wahl sind keine Legitimitätsressourcen, weder kann es sich auf einen demos stützen (Peter Gauweiler 2008b, 16611A) noch ist es gleichheitsgerecht gewählt und somit nicht legitimiert, „politische Leitentscheidungen zu treffen, die in einer Demokratie repräsentativ und zurechenbar sein müssen" (Peter Gauweiler 2009a, 25582B; vgl. Henry Nitzsche 2008b, 16472Bf.). Die nationale Wahl bleibt maßgebender Input-Legitimationsmodus. Damit zusammenhängend muss die Ausgestaltung des Throughput es zulassen, Entscheidungen auf die Bürger der Mitgliedstaaten (also nicht das Unionsvolk) zurückzuführen.

Vor allem Peter Gauweiler bemüht formal-juristische Argumente: Input und Throughput müssen die nationale Legitimationskette zum Tragen kommen lassen und die Abtretung legislativer Zuständigkeiten des Bundestags darf nicht überdehnt werden. ${ }^{20}$ Begrenzte Ein-

$15 \mathrm{Zu}$ den Dimensionen vgl. stellvertretend auch Dieter Nohlen / Rainer-OlafSchultze / Suzanne Schüttemeyer (Hrsg.), Lexikon der Politik, Bd. 7: Politische Begriffe, München 1998, S. 348 ff.; Manfred G. Schmidt, Wörterbuch zur Politik, Stuttgart 2004, S. 412 f.; David Easton, A Systems Analysis of Political Life, New York / London / Sidney 1965, S. 157 f., S. 273; Seymour Martin Lipset, Political Man. The Social Bases of Politics, London 1983, S. 64 ff.; Fritz W. Scharpf, a.a.O. (Fn. 5).

16 Vgl. David Beetham / Christopher Lord, Legitimacy and the EU, London 1998; Michael Zürn, Regieren jenseits des Nationalstaats. Globalisierung und Denationalisierung als Chance, Frankfurt am Main 1998.

17 Insgesamt bestand das Codierschema aus 238 Codes. Es wurde immer auf der untersten möglichen Ebene codiert, folglich dienten einige Codes/Oberkategorien zuvorderst der Strukturierung. Nach Abzug dieser verbleiben noch 189 Codes, die tatsächlich vergeben wurden.

18 Hierunter fallen Peter Gauweiler (CSU), Henry Nitzsche (fraktionslos) und Hermann Scheer (SPD).

19 Fritz W. Scharpf, a.a.O. (Fn. 5), S. 168.

20 Vgl. Dieter Grimm, Europas Verfassung, in: Gunnar Folke Schuppert / Ingolf Pernice / Ulrich Haltern (Hrsg.), Europawissenschaften, Baden-Baden 2005, S. 177 - 200. 
zelermächtigung, Mitgliedstaaten als „Herren der Verträge“ und Subsidiaritätswahrung werden akzentuiert. In der Forderung nach Einstimmigkeit tritt die Bedeutung der national vermittelten Legitimation abermals hervor. So könnten bei Nichtbeachtung „die auf europäischer Ebene getroffenen Entscheidungen [...] nicht mehr hinreichend von den Völkern der Mitgliedstaaten - über deren Regierungsvertreter im Rat - legitimiert werden " (Peter Gauweiler 2008b, 16610D). Offenbar ist Regieren auf europäischer Ebene nur legitim, solange die intergouvernementale Legitimation lückenlos bleibt. ${ }^{21}$ Auch Henry Nitzsche (2008b, 16472Bf., näher dazu unten) bezieht sich auf parlamentarische Beteiligung, zumal er eine Dominanz der Exekutive und ihre Übernahme legislativer Kompetenzen als legitimatorisches Hindernis ausmacht.

Dies verweist bereits auf die perzipierten Auswirkungen auf die nationale Ebene: Gegenwärtig würden Souveränität und demokratische Qualität, somit auch Legitimität und Wahrung des Grundgesetzes aufs Spiel gesetzt (Peter Gauweiler 2008b, 16610Cf.). ${ }^{22}$ Die Legislativfunktion des Bundestags würde mit Kompetenzübertragungen - durch legislative Europäisierung $^{23}$ - zu sehr geschwächt (Peter Gauweiler 2008b, 16610B). Ähnlich warnt Nitzsche (2009c, 26353C) die Abgeordneten vor Kompetenzabtretungen, würden sie doch „[etwas] verschenken [...], das [i] hnen vom deutschen Volk nur geliehen war“. Die Volkssouveränität könne nicht durch parlamentarisches Ratifikationsgesetz, sondern nur vom Souverän, dem Volk, durch einen verfassunggebenden Akt abgetreten werden (Peter Gauweiler 2008b, 16610C). ${ }^{24}$ Beide fordern die Durchführung von Referenden zur Ratifikation, wobei die Motivation bei Nitzsche (2009c, 26353Cf.; 2008b, 16472B) nicht zuletzt in einer Unzufriedenheit mit dem Funktionieren der Demokratie, auch in der Bundesrepublik Deutschland, liegt, während Gauweiler (2008b, 16610Cf.) Legalitätsargumente anbringt. Folglich ist zumindest bei Letzterem die Existenz eines demos der Errichtung des kratos und damit der genuin europäischen Legitimitätserzeugung vorgelagert. ${ }^{25}$ Bei Nitzsche scheint die Ablehnung der Ausgestaltung der europäischen Ordnung und der Auswirkungen auf die deutsche (auch) einer nationalistischen Idee zu entspringen:

„Künftig wird die für Deutschland entscheidende Politik von 27 Staats- und Regierungschefs bestimmt, von denen mindestens 26 nicht deutsch sind. Wie sich das mit dem Leitsatz aus Art. 20 Abs. 2 des Grundgesetzes [...] verträgt, ist mir schleierhaft" (Henry Nitzsche 2008b, 16472C).

Zwar argumentiert er unter Rückgriff auf Bestimmungen des GG, verwebt dann aber demos und ethnos, politische mit kultureller beziehungsweise ethnischer Identität. ${ }^{26}$ In der Konse-

21 Angesichts der geringen Relevanz der Output-Legitimation und der Bedeutung nationaler Legitimationsketten als Input bricht das Spannungsverhältnis zwischen Einstimmigkeit und Effizienz nicht auf. Vgl. Robert A. Dahl, A Democratic Dilemma. System Effectiveness versus Citizen Participation, in: Political Science Quarterly, 109. Jg. (1994), H. 1, S. 23 - 34.

22 Vgl. Claus Offe / Ulrich K. Preuss, The Problem of Legitimacy in the European Polity: Is Democratization the Answer?, in: Colin Crouch / Wolfgang Streeck (Hrsg.), The Diversity of Democracy. Corporatism, Social Order and Political Conflict, Cheltenham / Northampton 2006, S. 175 204, S. 177.

23 Vgl. Annette Elisabeth Töller, a.a.O. (Fn. 8).

24 Vgl. Ernst-Wolfgang Böckenförde, Demokratie als Verfassungsprinzip, in: Josef Isensee / Paul Kirchhof (Hrsg.), Handbuch des Staatsrechts der Bundesrepublik Deutschland, Bd. II: Verfassungsstaat, Heidelberg 2004, S. 429 - 496, S. $432 \mathrm{ff}$.

25 Vgl. Peter Graf Kielmansegg, a.a.O. (Fn. 5), S. 57.

26 Die EU wird unter anderem als „Milliardengrab für uns Deutsche“, als „,neues Versailles für 
quenz wird europäisches Regieren als Fremdbestimmung wahrgenommen (Henry Nitzsche 2008b, 16472B). ${ }^{27}$

Auch Hermann Scheer (2009c, 26372B) befürchtet den „Verlust an demokratischer Entscheidungskompetenz auf einzelstaatlicher, regionaler und kommunaler Ebene“; dies aufgrund der Überzeugung, ein politisches System sei mit zunehmender Größe weniger in der Lage, die Forderungen der Bürger im politischen Prozess zu verarbeiten. ${ }^{28}$ Nicht zuletzt wird mit Dezentralität die Unterstützung der Bürger in Verbindung gebracht, und auch die Betonung der Subsidiarität ist in diese Logik einzuordnen (Hermann Scheer 2009c, 26372Bf.). Ähnlich ist das Idealbild der Prozessebene hierauf bezogen:

„Es geht also nicht um einen Gegensatz von nationalen und europäischen Entscheidungskompetenzen [...], sondern um prinzipielle Fragen der Demokratie, die möglichste Transparenz, Überschaubarkeit, Unmittelbarkeit und Nähe der gewählten Repräsentanten verlangt" (Hermann Scheer 2009c, 26372B).

Offenbar sieht Scheer (2009c, 26372C) keine „demokratische Sensibilität“ im europäischen Regieren, das durch „offenkundige Entfremdungen zwischen Repräsentierten und Repräsentanten" gekennzeichnet sei.

Hinsichtlich der Parlamentsfunktion zielen alle auf Kontrolle. Scheer (2009c, 26372A) akzentuiert die Sekundärrechtsetzung und die Mitwirkung des Bundestags, vor allem zur Kontrolle der Subsidiaritäts- und damit der Kompetenzeinhaltung. Bei Gauweiler und Nitzsche sind die Kontrolle der Kompetenzabtretung sowie die Wahrung eigener Zuständigkeiten in der Primärrechtsetzung vordergründig. In einer Art „Souveränitätsreflex “29 soll der legislativen Europäisierung und der Entleerung der nationalen Demokratie Einhalt geboten werden (Henry Nitzsche 2009c, 26353C; Peter Gauweiler 2009a, 25583C). Ähnlich mahnt Nitzsche einen Wandel im Selbstverständnis des Parlaments an und fordert eine vermehrte Kontrolle der Regierung. Insbesondere der Duktus verweist auf die Demokratieunzufriedenheit; so hätten „wir es in Deutschland nicht mit freien Abgeordneten zu tun [...], sondern mit willfährigen Parteisklaven“" (Henry Nitzsche 2009c, 26353D).

Vor der Folie des jeweiligen Legitimitätsdenkens besteht ein plausibler Zusammenhang: Gauweiler und Nitzsche gehen von einer grundsätzlichen Demokratieunfähigkeit der EU aus, Scheer attestiert besseres legitimatorisches Potenzial unterer Ebenen. Während Erstere darauf bedacht sind, der EU keine überbordenden Kompetenzen zukommen zu lassen, die demokratische Substanz der nationalen Ebene zu wahren und dafür zu sorgen, dass diese beziehungsweise der Bundestag das Heft in der Hand behält, sind es bei Scheer gerade die Prozess-Dimensionen, die auf europäischer Ebene nicht optimal sind.

Deutschland“ und der Vertrag von Lissabon als „neuerliches Ermächtigungsgesetz“, durch das „Brüssel [legitimiert werde, Anmerkung der Verfasserin] allmächtig und ungehindert über deutsche Interessen zu entscheiden" (Nitzsche 2008b, 16472D) bezeichnet. Vgl. Hans Joas / Christof Mandry, Europa als Werte- und Kulturgemeinschaft, in: Gunnar Folke Schuppert / Ingolf Pernice I Ulrich Haltern (Hrsg.), a.a.O. (Fn. 20), S. 541 - 572, S. 569.

27 Vgl. Peter Graf Kielmansegg, a.a.O. (Fn. 5), S. 57.

28 Vgl. Robert A. Dahl, a.a.O. (Fn. 21), S. 28.

29 Der Begriff entstammt Andreas Hofmann / Wolfgang Wessels, Der Vertrag von Lissabon - eine tragfähige und abschließende Antwort auf konstitutionelle Grundfragen?, in: Integration, 31. Jg. (2008), H. 1, S. 3 - 20, S. 6. Sie beziehen sich auf die Kompetenzbegrenzung des Lissabon-Vertrags, um die de jure Souveränität der Mitgliedstaaten nicht einzuschränken. 


\subsection{Reformpotential, Kontrolle und Kompetenzwahrung}

Eine zweite Gruppe von MdBs, die empirisch eine ablehnende Position einnimmt, sieht „Reformpotential in allen Variablen“30. Die Output-Dimension ist legitimationsrelevant, allerdings führt der Abgleich der eigenen Wert- und Zieldefinition mit Politikergebnissen und vertraglichen Zielsetzungen zu einer ablehnenden Haltung und der Wahrnehmung eines Legitimitätsmangels:

„Wir sind stolz darauf, dass wir, die Linke, nicht Ja sagen zu einem Europa, in dem die Armut wächst, in dem die Schere zwischen Arm und Reich immer weiter auseinandergeht" (Alexander Ulrich 2008a, 15856B).

Das Legitimitätsdefizit liegt in einem „basic ideological disagreement“31 über inhaltliche Ziele und Leistung der EU, dem perzipierten Missverhältnis zwischen (neo-)liberalem und sozialem Output. ${ }^{32}$ Für Jörg Tauss (2009b, 26271Af.) ist die Problematik im Output die mangelnde Gewährleistung von Freiheit und Bürgerrechten, die der Sicherheitsorientierung weichen müssen.

Input und Throughput dienen dem Erzielen inhaltlich anerkennungswürdiger Politikergebnisse, was bei Dieter Dehm mit der absoluten Zentralität der Output-Variable besonders hervortritt. Für Lothar Bisky und Gregor Gysi haben In- und Throughput einen vergleichsweise stärkeren normativen Eigenwert. Letzterer wolle

„die europäische Integration wirklich nicht weniger als Sie. [...] Ich weiß, was das für den Frieden bedeutet. Aber ich sage Ihnen: Das geht niemals angesichts der Ängste, die gegenwärtig herrschen. Wir müssen die Bürgerinnen und Bürger mitnehmen [...]“ (Gregor Gysi 2009b, 26259C; vgl. Lothar Bisky 2008b, 16460C).

Die Ausgestaltung von In- und Throughput, also Beteiligungsmöglichkeiten, Responsivität der politischen Akteure und Entscheidungen durch Bürger, ist zentral für deren empirische Akzeptanz (Gregor Gysi 2009b, 26260B). So fordern Ulrich, Gysi und Bisky (Dehm am Rande) die Abhaltung von Referenden bei Vertragsänderungen. „Europa [müsse] auf der Zustimmung seiner Menschen aufbauen“ und die Bürger „ihr Europa mitgestalten“ (Lothar Bisky 2008a, 15842A; 2008b, 16460D). Ulrich (2008a, 15848C) artikuliert mit Blick auf die negativen Referenden zum Verfassungsvertrag, die Bürger wollten „zwar eine Verfassung, aber nicht Sozialabbau, Aufrüstung und eine neoliberale Wirtschaftsordnung mit Verfassungsrang". So ist die Forderung nach Referenden neben dem Input-Erfordernis der Bürgerbeteiligung mit der Gestaltung der vertraglichen Zielsetzungen und einem responsiven Politikprozess verbunden. ${ }^{33}$ Mit Blick hierauf werden die Reformen in der parlamentari-

30 Es sind Lothar Bisky, Dieter Dehm, Alexander Ulrich (alle Die Linke) und Jörg Tauss (fraktionslos) subsumiert. Tauss war zur Abstimmung am 24. April 2008 Mitglied der SPD-Fraktion, gehörte dieser aber zum Zeitpunkt der analysierten Rede nicht mehr an.

31 David Beetham / Christopher Lord, a.a.O. (Fn. 16), S. 24.

32 Vgl. zur Asymmetrie von negativer und positiver Integration Fritz W. Scharpf, a.a.O. (Fn. 5). Übrigens beziehen sich alle Redner der Linken auch auf das Richterrecht des EuGH, das Liberalisierung zulasten sozialer Ziele überprivilegiere (Lothar Bisky 2008b, 16462Df.; Dieter Dehm 2009b, 26268Bf.; Gregor Gysi 2009b, 26260B; Alexander Ulrich 2009c, 26352A). Vgl. Fritz W. Scharpf, Legitimität im europäischen Mehrebenensystem, in: Leviathan, 37. Jg. (2009), H. 2, S. $244-280$.

33 Diese Argumentation trägt andere Züge als jene Peter Gauweilers, der Legitimation, sofern bestimmte Grenzen überschritten werden, nur durch einen verfassungsgebenden Akt für möglich hält. Während hier offenbar legitime „Staatsgewalt“ auf EU-Ebene „im Interesse des Volkes und 
schen Beteiligung „[als] erste Schritte zu mehr direkter Demokratie“ (Lothar Bisky 2008b, 16461D) gewertet und die legitimationshemmende Dominanz der Exekutiven kritisiert:

„Bei der Vorratsdatenspeicherung hat der Deutsche Bundestag [...] gesagt: Wir wollen das nicht. - Daraufhin ging die Exekutive [...] nach Brüssel und hat dort den Beschluss dieses Parlamentes [...] ausgehebelt. [...] Es gibt weitere Beispiele [...]“ (Jörg Tauss 2009b, 26270Df.; vgl. Lothar Bisky 2008a, 15842Af.; Alexander Ulrich 2009c, 26352B).

Die nationale Exekutive, zumal legislative Funktionen übernehmend und die Gewaltenteilung verkehrend, finde auf europäischer Ebene eine Exit-Option, um den Willen des Parlaments zu unterwandern. Für Tauss (2009b, 26271A) ist weiter die damit verbundene Intransparenz des Politikprozesses heikel. Daraus ergeben sich nationale Legitimitätsprobleme: Einerseits schwänden dem Bundestag Legislativkompetenzen, gleichzeitig laufe man Gefahr, die Verfassung zu verletzen, denn „,[d]ie Väter und Mütter des Grundgesetzes haben Grundrechte und Freiheit vorangestellt; sie haben [...] kein Grundrecht auf Sicherheit geschaffen“. Durch das ,Spiel über Bande` würde diese Priorisierung verkehrt und die Exekutive könne „in Europa die Axt an unsere Verfassung legen“ (Jörg Tauss 2009b, 26271B). ${ }^{34}$

Ähnlich wie Gauweiler argumentiert Gysi (2009a, 25578A) unter Berufung auf das Bundesverfassungsgericht: Um die Legitimität der Bundesrepublik nicht zu gefährden, müsse den Mitgliedstaaten hinreichender Gestaltungsraum verbleiben und der Vertrag von Lissabon sei nur in der Interpretation des Bundesverfassungsgerichts mit dem GG vereinbar (Gregor Gysi 2009a, 25577Cf.). Offenbar ist es von enormer legitimatorischer Bedeutung, das Letztentscheidungs- beziehungsweise Letztinterpretationsrecht im nationalen Raum zu wahren.

Hierauf deuten auch die Parlamentsfunktionen, die vor allem in der nationalen Kompetenzwahrung liegen (zum Beispiel Lothar Bisky 2008b, 16461C; Alexander Ulrich 2009c, 26351B). Es stehen Bestimmungen im Zentrum, die im Vertragstext zweideutig geregelt und potentiell geeignet erscheinen, gegen das Grundgesetz zu verstoßen (Gregor Gysi 2009a, 25577Cf.; Dieter Dehm 2009a, 25584A). Auch hätten Stellungnahmen des Bundestags gegenüber der Regierung generell verbindlich zu sein (zum Beispiel Gysi 2009b, 26259Cf.). Ähnlich kompetenzwahrend argumentiert Dehm (2009b, 26268A) und verlangt für die Gemeinsame Außen- und Sicherheitspolitik sowie die Sicherheits- und Verteidigungspolitik weitergehende Regelungen im Begleitgesetz, wohl als Basis einer Kontrollermöglichung. Auch Tauss (2009b, 26271A) bezieht sich auf das Verhältnis Parlament - Regierung, denn es sei

„eine Frage des Selbstbewusstseins dieses Parlaments, ob es sich so etwas [die Umgehung des Bundestags, Anmerkung der Verfasserin] auch künftig gefallen lässt oder nicht. [...] Wenn wir kein parlamentarisches Rückgrat gegenüber der Exekutive haben, im Zweifel über Fraktionsgrenzen hinweg, dann werden einige Damen und Herren [... ] weiterhin mit uns Jojo spielen“.

Das Parlament müsse die Regierung inhaltlich, im Sinne einer politischen Richtungskontrolle, überwachen. Hier spiegelt sich der Verdruss hinsichtlich des ,Spiels über Bande', das verantwortlich für den als illegitim angesehenen Output zeichnet. Die insgesamt kontrollierenden Funktionen zielen darauf, das Parlament vor Kompetenzübertragung oder (schleichendem) Kompetenzverlust zu schützen, wobei vor der Folie des Legitimitätsdenkens an den jeweiligen neuralgischen Punkten des perzipierten Defizits angesetzt wird.

zu seinem Wohl ausgeübt" werden soll, dominiert bei Gauweiler „die Trägerschaft der Staatsgewalt" (Ernst-Wolfgang Böckenförde, a.a.O. (Fn. 24), S. 432).

34 Vgl. Klaus von Beyme, a.a.O. (Fn. 8), S. 26; Tapio Raunio / Simon Hix, a.a.O. (Fn. 8), S. 142 ff. 


\subsection{Duale Legitimation, anerkennungswürdiger Output und gestaltende Beteiligung}

Das dritte - empirisch zustimmende - Legitimitätskonzept speist sich aus dualer Legitimation und inhaltlicher Output-Dimension. ${ }^{35}$ Die parlamentarische Legitimation über nationale Parlamente und EP wird als Stärkung der Demokratie und/oder Legitimität bezeichnet (zum Beispiel Carl-Christian Dressel 2008a, 15853A; Thomas Silberhorn 2008a, 15854C; Jürgen Trittin 2008b, 16463C). Hinsichtlich der Rolle des EP heben sich die MdBs teils explizit von den Setzungen des Bundesverfassungsgerichts ab (zum Beispiel Michael Roth 2009c, 26357A), denn der Lissabon-Vertrag würde „die Demokratisierung durch die Parlamentarisierung durchsetzen“ (Axel Schäfer 2008b, 16475C). Die EP-Wahlen erscheinen als zentraler Legitimationsmechanismus (Michael Roth 2009a, 25581C), und durch Responsivität sowie direkte Bürgerbeteiligung wird die Demokratie als gestärkt empfunden (zum Beispiel Carl-Christian Dressel 2009a, 25585C; Hans-Christian Ströbele 2008b, 16614C).

Nicht zuletzt speist sich die Legitimität aus der Output-Leistung; so könne „das Vertrauen der Menschen nur [gewonnen werden], wenn sie Europa als Schutzmacht ihrer ganz persönlichen Interessen erleben“" (Rainder Steenblock 2008b, 16470C; vgl. Michael Roth 2008a, 15844C). Offenbar wird davon ausgegangen, dass bei den Bürgern aus einer spezifischen eine diffuse Unterstützung und Vertrauen heranwachsen könne. ${ }^{36}$ Gunther Krichbaum (2008a, 15847D) formuliert, es gebe „[...] Dinge, die wir dem Bürger begreiflich machen müssen; denn das verdeutlicht jedem, dass er die Existenz und Sicherung des eigenen Arbeitsplatzes auch Europa zu verdanken hat".

$\mathrm{Zu}$ den Politikergebnissen zeigt sich eine unterschiedlich kritische Beziehung. Im Großen und Ganzen aber werden bisheriger Output und Zieldefinitionen des Lissabon-Vertrags unterstützt. Besondere Bedeutung kommt, meist bei Rednern der SPD und der Grünen, der sozialen Dimension zu, speziell hinsichtlich der Zustimmung der Bevölkerung und der Responsivität (zum Beispiel Angelica Schwall-Düren 2008b, 16465A; Rainder Steenblock 2008a, 15843Cf.). Wenngleich er den Output weitgehend positiv evaluiert, bewertet Markus Löning (2008a, 15839C) vor liberalen Idealen die Vertragsziele gegenteilig und kritisiert,

„dass soziale Ziele, die immer staatliches Handeln nach sich ziehen, nach vorne gerückt sind, während der Wettbewerb abgestuft ist [...]. Ein Grundwert Europas ist immer gewesen, dass die Freiheit des Einzelnen zählt".

Ähnlich rügt Roth (2008a, 15845B) das aus der Sicherheitsfokussierung resultierende Ungleichgewicht im Raum der Freiheit, der Sicherheit und des Rechts (RFSR). Teils werden identifizierte Missstände im Output auf bundesrepublikanische Regelungen (zum Beispiel Jürgen Trittin 2008b, 16463A) oder eine unrichtige Darstellung im nationalen Diskurs zurückgeführt. Demgemäß steht die normative Anerkennungswürdigkeit europäischen Regierens nicht in Frage; maximal besteht ein - national zu verantwortendes - Akzeptanzproblem:

35 Hierunter fallen Carl-Christian Dressel, Michael Roth, Axel Schäfer, Angelica Schwall-Düren (alle SPD), Gunther Krichbaum (CDU), Markus Löning (FDP), Thomas Silberhorn (CSU), Rainder Steenblock, Hans-Christian Ströbele und Jürgen Trittin (alle Die Grünen).

36 Vgl. stellvertretend David Easton, a.a.O. (Fn. 17), S. 157 f., S. 273; sowie den Überblick bei Susanne Pickel / Gert Pickel, Politische Kultur- und Demokratieforschung. Grundbegriffe, Theorien, Methoden. Eine Einführung, Wiesbaden 2006, S. 78 ff. 
„Das ist ein Punkt [Regelungen zur Antidiskriminierung, Anmerkung der Verfasserin], an dem Sie [Volker Kauder, Anmerkung der Verfasserin] Europa nicht nur schlecht verkaufen, sondern den Gedanken auf den Kopf stellen [...]“ (Jürgen Trittin 2008a, 15850C). ${ }^{37}$

Eine diffuse Unterstützung des Integrationsprozesses kommt vielfach in dem Wunsch zum Ausdruck, die Ratifikation solle Signalwirkung entfalten „innerdeutsch wie auch innereuropäisch; denn wir müssen zeigen, dass wir für die europäische Einigung einstehen " (CarlChristian Dressel 2009b, 26272B; vgl. Angelica Schwall-Düren 2009c, 26354C). Mit dem Bezug auf die Präambel des Grundgesetzes rechtfertigt Schäfer (2008b, 16476B) die Integration historisch und bezieht vor diesem Hintergrund Stellung zum Urteil des Bundesverfassungsgerichts:

„Wir schauen in die Begründung, die Karlsruhe gegeben hat. Da wird 33-mal die nationale Souveränität betont. [...]. Die klugen Väter und Mütter unserer Verfassung [...] haben diesen Begriff in dieser Konfrontation bewusst nie verwendet. [...] Sie haben [...] gesagt: Deutschland will und Deutschland soll nie mehr so souverän sein, dass es in der Lage ist, andere Länder mit Krieg zu überziehen“ (Axel Schäfer 2009b, 26262Bf.).

Mit der Kriegserinnerung wird auf die Unfähigkeit der Nationalstaaten, Sicherheit zu gewährleisten ${ }^{38}$, verwiesen und der Friede in Europa betont.

Häufig werden die Zielsetzungen des Lissabon-Vertrages als Widerspiegelung geteilter Werte aufgefasst (Carl-Christian Dressel 2008a, 15852D; Markus Löning 2008a, 15839C), was auf eine Konzeption der EU (auch) als Wertegemeinschaft verweist. ${ }^{39}$ An konstitutive Werte bindet auch Roth (2008b, 16471Bf.) Output-Zieldefinition und auswärtiges Handeln der Union: Dieses habe sich an der Logik der Angemessenheit ${ }^{40}$ zu orientieren, um als legitim gelten zu können. Außerdem macht er (2009a, 25581C) in der Vielfalt der EU27 eine potentielle Gefährdung der Handlungs- und Entscheidungsfähigkeit - und damit der Legitimation über den Output - aus. Thomas Silberhorn (2008a, 15853Df.) konzipiert Parlamente als Bindeglieder, die das Dilemma aus Systemeffektivität und Bürgerbeteiligung zügeln. ${ }^{41}$ Diese Verwebung von Through- und Output akzentuiert auch der Verweis auf das Potential der institutionellen Reformen:

„Ich kenne kaum jemanden in Deutschland, der mir erklären kann, wie der Motor eines Autos funktioniert. Die meisten setzen sich ins Auto und wollen damit fahren. So ist es auch mit Europa" (Gunther Krichbaum 2008a, 15847B; vgl. Markus Löning 2009c, 26349B).

Mit ähnlicher Stoßrichtung wird das Bundesverfassungsgericht angeprangert. So habe

„[d]iese nationale Souveränität, von der das Bundesverfassungsgericht spricht, [...] mit dem 21. Jahrhundert und den Herausforderungen der Globalisierung nichts zu tun. [...] Wir sind zur europapolitischen Zusammenarbeit verpflichtet“" (Michael Roth 2009c, 26357B).

37 Ähnlich argumentieren Trittin (2008a, 15850Af.) und Steenblock (2008b, 16470D) hinsichtlich des Throughput. Krichbaum (2009c, 26350Df.) verbindet eine Würdigung der Arbeit des EP mit der Forderung, sie national besser zu kommunizieren.

38 Vgl. David Beetham / Christopher Lord, a.a.O. (Fn. 16), S. 124.

39 Vgl. Armin von Bogdany, Europäische Verfassung und europäische Identität, in: Gunnar Folke Schuppert / Ingolf Pernice / Ulrich Haltern (Hrsg.), a.a.O. (Fn. 20), S. 331 - 371, S. 352.

40 Vgl. James G. March / Johan P. Olsen, The Logic of Appropriateness. Arena Working Paper 04/09, Oslo 2004.

41 Vgl. Robert A. Dahl, a.a.O. (Fn. 21), S. 32 - 33. 
Offensichtlich wird politisches Gestaltungspotential in einigen Politikbereichen mehr auf europäischer als auf nationaler Ebene gesehen (zum Beispiel Gunther Krichbaum 2009c, 26350D; Axel Schäfer 2009b, 26263Bf.; Jürgen Trittin 2008a, 15851Cf., näher dazu unten).

Gestalterisch-mitwirkende Funktionen dominieren dann auch bei der Positionierung des Bundestags. Einschätzungen, wie er solle seiner „Verpflichtung gerecht werden“ (Axel Schäfer 2009b, 26263D; vgl. zum Beispiel Carl-Christian Dressel 2009b, 26272C), sprechen für die Perzeption einer Verantwortung für vertiefte Integration. Etwas weniger proaktiv denkt Roth (2008b, 16471D), dessen Verweis auf sensible Bereiche auf stärkere Kontrolle schließen lässt und vor der Folie der kritischen Haltung zum RFSR zu lesen ist. Auch sei der Bundestag in der Sekundärrechtsetzung „dazu aufgerufen, auf der demokratischen Seite mehr zu machen, die neue Bundesregierung besser zu kontrollieren“, schon um sich nicht „völlig unglaubwürdig“ (Markus Löning 2009c, 26349C, 26349B) zu machen, womit neben der Legitimität des Bundestags auf die Abwehr einer legislativen Europäisierung verwiesen ist. Dennoch zielt die Positionierung meist auf inhaltliche Gestaltung oder ist als frühzeitig mitgestaltende Kontrolle zu charakterisieren. Es sei

„zentrale Aufgabe des Deutschen Bundestages [...], Regierungshandeln innerstaatlich zu kontrollieren. Die Bundesrepublik Deutschland wird im Rat von der Bundesregierung vertreten. Hier müssen wir kontrollieren und versuchen, Einfluss zu nehmen" (Michael Roth 2008a, 15845C; vgl. Axel Schäfer 2009a, 25572D).

Intendiert ist, „sich frühzeitig einzuschalten und auf die europäische Rechtsetzung Einfluss zu nehmen" (Hans-Christian Ströbele 2008b, 16613Df.), die Kontrolle der Regierung zu nutzen, um gestalterischen Einfluss auf die europäische Rechtsetzung zu erlangen und die Regierung ex ante zu steuern.

Auch dürfe das Parlament „mit der Keule der Subsidiaritätskontrolle oder -rüge nicht all das, was uns möglicherweise politisch missfällt, zerschlagen“, sondern müsse „dafür sorgen, dass es [...] keine neuen Blockaden gibt, sondern konstruktive Mitgestaltung“" (Michael Roth 2008a, 15845Cf.; vgl. Jürgen Trittin 2008a, 15850B), offenbar um Handlungsfähigkeit zu gewährleisten. Thomas Silberhorn verknüpft die Subsidiaritätskontrolle mit der Kritik an der Exekutivlastigkeit und dem inhaltlichen Handeln der Ratsvertreter und fordert, dass auch die supranationalen Institutionen mögliche Konflikte zwischen Regierungshandeln im Rat und Parlamentswillen erkennen müssten. Dazu sei „die Wahrung der Handlungsspielräume der nationalen Parlamente unsere Aufgabe als Wächter des Subsidiaritätsprinzips“ (Thomas Silberhorn 2008a, 15854C). In Steenblocks (2008b, 16470Af.) Lesart ermöglicht die Subsidiaritätsklage den Parlamenten mehr Gestaltung, und der Bundestag müsse

„[...] sehr selbstbewusst sagen: Wir arbeiten. Wir können die Regierung kontrollieren. Wir können die Entscheidung auf europäischer Ebene mitgestalten [...]. Dahinter stehen muss das Selbstbewusstsein zu sagen: Wir stehen hier als diejenigen, die gewählt worden sind - mit Rechten und Pflichten" (Rainder Steenblock 2009a, 25580C).

Der Rekurs auf die nationale Wahl verweist auf die Herleitung der „Ausübung der Staatsgewalt ihrem Inhalt nach vom Volk ${ }^{“ 42}$ - die Repräsentationsfunktion. ${ }^{43}$ So müssten die Parlamentarier Bevölkerungsinteressen vermitteln und „in den Kontakt mit den Bürgerinnen

42 Ernst-Wolfgang Böckenförde, a.a.O. (Fn. 24), S. 441.

43 Auch habe der Bundestag aufgrund der direkten Legitimation die Einschätzungsprärogative und die Verantwortung für die Ausgestaltung der Integration - nicht das BVerfG (Gunther Krichbaum 2009c, 26350Af.). 
und Bürgern unseres Landes treten [...], um Europa dichter an die Menschen zu bringen“ (Rainder Steenblock 2009a, 25579A; vgl. zum Beispiel Gunther Krichbaum 2008a, 15848A). Insofern können diese Ausführungen als aktive Rolle zur Akzeptanzsteigerung - zumal national unrichtige Kommunikation konstatiert wurde - gedeutet werden.

Dennoch wird meist ein Bedarf an weiteren Regelungen erkannt, die als Forderung nach aktiver Europäisierung ${ }^{44}$ gelten können (zum Beispiel Angelica Schwall-Düren 2009c, 26355A; Jürgen Trittin 2008a, 15850B). Steenblock (2009b, 26260D; vgl. zum Beispiel Roth 2009a, 22581A) betitelt das Urteil des Bundesverfassungsgerichts als einen „Tritt vor das Schienbein“, denn der Bundestag hätte das „selber schon vorher regeln können“. Dieses Versäumnis führt er auf „ein Problem unseres Selbstverständnisses“ zurück. Stattdessen müssten „Parlamentarier solche Strukturen schaffen“ (Rainder Steenblock 2009a, 25580Af.), die es ermöglichen, die Regierung zu kontrollieren und mit nationalen Parlamenten sowie EP in einen Diskussionsprozess einzutreten. Mithin sind zumindest einige dieser Parlamentarier als „euro-wizards “ 45 zu bezeichnen, die durch eigene Initiativen versuchen, europäische Politik zu beeinflussen, und Aufmerksamkeit für ihre Belange von europäischen Entscheidungsträgern fordern.

\subsection{Nutzung neuer Handlungsspielräume zur Beantwortung globaler Herausforderungen}

Die bereits angedeutete Handlungs- und Problemlösungsfähigkeit erhält besondere Bedeutung, wenn die EU ihre Legitimität durch die „Antwort auf globale Herausforderungen“ schöpft. ${ }^{46}$ Für Michael Link und Florian Toncar ist es im Politikprozess wichtig, das Subsidiaritätsprinzip einzuhalten, nicht zuletzt wegen der Vielfalt der EU und der Heterogenität der Gesellschaft. So sei „eine sinnvolle Abgrenzung“ der Kompetenzen eine „Schlüsselfrage bei der Weiterentwicklung der Europäischen Union“" (Florian Toncar 2008a, 15846D). Es wird dafür plädiert, dass der EuGH- ähnlich einer Konfliktschlichtungsinstanz zwischen föderalen Ebenen ${ }^{47}$ - das Subsidiaritätsprinzip verteidigen müsse (Michael Link 2008b, 16466Af.; Florian Toncar 2008a, 15847A). Im Output stehen ökonomische Leistung, freier Wettbewerb und Wettbewerbsfähigkeit im Fokus, ein Legitimitätsdenken in liberal-freiheitsschaffender Tradition ist erkennbar. Bei Toncar (2008a, 15846Af.) tritt Freizügigkeit hinzu:

„Europa ist ein Projekt der Freiheit. Grenzen fallen [...]. Europa stärkt nämlich nicht nur unsere Wettbewerbsfähigkeit [...], sondern Europa fördert insgesamt die gegenseitige Toleranz von Menschen [...]. Ich glaube, dass diese Fähigkeit [...] eine der Schlüsselqualifikationen im Zusammenhang mit der Globalisierung insgesamt ist“"

Demgegenüber richten die übrigen Redner den Blick auf den Zugriff auf übernationale Problemlagen durch beziehungsweise die mitgliedstaatliche Interessenvertretung über die EU und damit deren Beitrag zur Problemlösung (zum Beispiel Norbert Röttgen 2009b,

44 Vgl. Katrin Auel, a.a.O. (Fn. 1).

45 Katrin Auel / Arthur Benz, a.a.O. (Fn. 1), S. 386 - 387; vgl. Annette Elisabeth Töller, a.a.O. (Fn. 8).

46 Darunter fallen Michael Link, Florian Toncar (beide FDP), Thomas Oppermann (SPD), Norbert Röttgen, Andreas Schockenhoff, Michael Stübgen (alle CDU) und Manuel Sarrazin (Die Grünen).

47 Vgl. Rudolf Hrbek, Modelle politischer Ordnung. Föderalismus, Mehrebenensystem, variable Geometrie, in: Osteuropa, 54. Jg. (2004), H. 5/6, S. 87 - 104, S. 92. 
26257Af.; Michael Stübgen 2009a, 25577A). Zumeist geht dies einher mit der Betonung von Handlungsfähigkeit. So lehnt Thomas Oppermann (2009b, 26254Af.) eine Mandatierung des Ratsvertreters und einen völkerrechtlichen Vorbehalt ab, denn damit hätte

„Deutschland als größter Mitgliedstaat [...] den geringsten Einfluss auf Entscheidungen gehabt [...]: In schwierigen Situationen muss die Regierung Verhandlungen aktiv gestalten können [...]. [...] [S]tellen Sie sich einmal vor, alle 27 Mitgliedstaaten würden völkerrechtliche Vorbehalte geltend machen. [...] Dann hätten wir ein komplett zersplittertes europäisches Vertragsrecht und rechtlich gesehen ein babylonisches Europa“.

Die Begründung über die Wahrung der Rechtseinheit kann als Befürchtung resultierender free-riding Probleme ${ }^{48}$ und Ablehnung allzu flexibler Integration ${ }^{49}$ gelesen werden. Weiter steht hiermit der Output-Nutzen für die Unions- und vor allem die Bundesbürger in Zusammenhang (Oppermann 2009b, 26253B). Verknüpft ist Handlungsfähigkeit zudem mit Akteursqualität:

„Entweder sind wir in der Lage, bei der Bewältigung der globalen Herausforderungen unsere Interessen gemeinsam zu vertreten, indem wir handlungsfähiger werden, oder wir lassen uns von anderen vorgeben, welche Rolle wir zu spielen haben" (Andreas Schockenhoff 2008a, 15840A; vgl. Michael Stübgen 2008b, 16474Af.).

Aus Sicht dieser MdBs führt also nationalstaatlich-unilaterales Handeln „zu struktureller Fremdbestimmung“, während gemeinsames Handeln staatliche Selbstbestimmung durch Teilhabe an der Regelsetzung erweitert. ${ }^{50}$ Auch spielt das Potential für die Vertretung nationaler Interessen eine Rolle, denn „wir [brauchen] Europa [...], wenn wir die deutschen Interessen in der Welt angemessen vertreten wollen" (Thomas Oppermann 2009b, 26253Af.).

Offenbar wird der Nationalstaat nicht für alle Problemlagen als adäquate Problemlösungseinheit angesehen. Allerdings unterscheiden sich die gezogenen Konsequenzen: Während manche in Europa die gemeinsame Zukunft sehen oder eine Verbesserung der mitgliedstaatlichen Legitimität attestieren, reagieren andere mit der Anmahnung einer engen Kompetenzführung (zum Beispiel Andreas Schockenhoff 2008a, 15840B). Gerade durch die Zentralität von Problemlösungs- und Handlungsfähigkeit, engen Kompetenzen und weitgehendem Fehlen einer Partizipations- und Repräsentationsdimension erinnert die Denkweise an eine Delegationsbeziehung. Es werden Bereiche übertragen, die national nicht bearbeitbar sind und in denen Delegation Effizienz und Output-Legitimität erhöht. ${ }^{51}$

Michael Stübgen (2009a, 25576D) formuliert, Legitimation sei über „Rückkopplung mit den nationalen Parlamenten“ zu gewährleisten. So seien die verfassungsgerichtlich gesetzten Grenzen der Übertragung von Hoheitsrechten sowie Subsidiarität zu wahren und die Einzelermächtigung fundamental, um lückenlose demokratische Legitimation zu gewährleisten (Michael Stübgen 2009a, 25576A). Ähnlich bemüht er Legalitätsargumente, wenn er betont, die Mitgliedstaaten blieben

„Herren der europäischen Verträge. Die Europäische Union hat eben keine Kompetenzkompetenz. [...] Natürlich ist die Europäische Union eine Rechtsgemeinschaft. Aber sie kann

48 Vgl. James A. Carporaso, The European Union and Forms of State: Westphalian, Regulatory or Post-Modern?, in: Journal of Common Market Studies, 34. Jg. (1996), H. 1, S. 29 - 52, S. 41.

49 Vgl. Rudolf Hrbek, a.a.O. (Fn. 47), S. 101.

50 Jürgen Neyer, Das Recht auf Rechtfertigung und die Legitimität von Supranationalität, in: Berliner Debatte Initial, 21. Jg. (2010), H. 2, S. 17 - 28, S. 19.

51 Vgl. Giandomenico Majone, a.a.O. (Fn. 4), S. 28; Andrew Moravcsik, a.a.O. (Fn. 4). 
vollen demokratischen Standards nur zusammen mit dem Grundgesetz genügen“ (Michael Stübgen 2009a, 25577 A).

Im übrigen charakterisiert Stübgen (2009a, 25576D; 2008b, 16473D) die EU als „Staatenbund“ und „Völkerbund“, was abermals auf die abgeleitete Legitimation, wie für internationale Organisationen typisch, verweist. Ähnlich erfolgt in Röttgens Verständnis die InputLegitimation über die Mitgliedstaaten; dennoch seien

„Deutschland und Europa, der Nationalstaat und Europa [...] wechselseitig und - im Sinne des Wortes - existenziell voneinander abhängig. [...] Dieses Verhältnis ist dadurch gekennzeichnet, dass keiner ohne den anderen kann" (Norbert Röttgen 2009b, 26257A).

Der Nationalstaat brauche Europa im Hinblick auf die Problembearbeitung, umgekehrt sei aber auch die EU auf den Nationalstaat angewiesen, denn

„[d]ie europäische Integration ist ohne die Verwurzelung in Regionen, ohne die kulturelle Identität, die durch Nationen vermittelt wird, ohne die demokratische Auseinandersetzung und Legitimation, die in den Nationalstaaten, in den Mitgliedstaaten, stattfindet, nicht denkbar [...]“ (Norbert Röttgen 2009b, 26257Bf.).

Damit ist eine „Input-Output-Inkongruenz“ angesprochen ${ }^{52}$ : Die nationale Ebene stellt Kanäle für demokratische Teilhabe bereit, während auf EU-Ebene zumindest ein Gutteil der Handlungs- und Problemlösungsfähigkeit angesiedelt ist. Dies ist normativ offenbar unproblematisch, dringliche Legitimitätsdefizite werden nicht hervorgerufen. Vielmehr erscheint die Legitimitätsgenerierung symbiotisch. ${ }^{53}$ Verknüpft werden In- und Output über nationale Parlamente; so bilde zwar

„Europa den wichtigsten Teil bei der Gestaltung der Globalisierung. Das heißt aber auch, dass es bei diesem Teil der Gestaltung nicht zur Entparlamentarisierung kommen darf" (Norbert Röttgen 2009b, 26258B).

Die Beteiligung nationaler Parlamente stärke die Akzeptanz der Integration in der Bevölkerung (Norbert Röttgen 2009b, 26258A), deren Partizipationsmöglichkeiten ohnehin verbessert würden, denn

„[d] urch den Prozess der europäischen Integration und auch durch den Vertrag von Lissabon werden die Handlungsfähigkeit der Demokratie und damit die Möglichkeiten der Menschen, über demokratische Wahlen auf Politik Einfluss zu nehmen, gestärkt, weil übernationale Interessenfelder und übernationale Fragestellungen von Politik überhaupt belangbar werden“ (Manuel Sarrazin 2009c, 26353A).

Insofern gewinnt Legitimation über Wahl erst mit der Integration an Gehalt, denn die EU

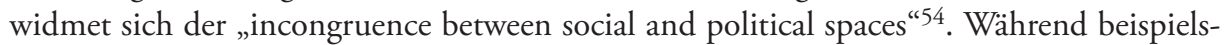
weise für Peter Gauweiler der Supranationalisierungsprozess ursächlich für die Einschränkung nationaler Handlungsfähigkeit und die Bedrohung des „government by the people“ ist, ist er hier die Reaktion auf beschränkte Möglichkeiten der Nationalstaaten und eine Zukunftsperspektive (Thomas Oppermann 2009c, 26348C).

52 Timm Beichelt, Deutschland und Europa. Die Europäisierung des politischen Systems, Wiesbaden 2009, S. 304; vgl. Fritz W. Scharpf, a.a.O. (Fn. 5), S. 12.

53 Vgl. Martin Höpner / Stephan Leibfried / Marcus Höreth / Fritz W. Scharpf / Michael Zürn, a.a.O. (Fn. 11), S. 349.

54 Michael Zürn, Democratic Governance Beyond the Nation-State: The EU and Other International Institutions, in: European Journal of International Relations, 6. Jg. (2000), H. 2, S. 183 221, S. 190. 
Inwiefern europäische und nationale Ebene als interdependent gedacht werden, ist auch für die Ausgestaltung der Parlamentsfunktionen verantwortlich. Die Positionierung des Bundestags folgt nicht - wie man intuitiv vermuten könnte - der Konzeption nationaler Legitimationsketten, wie sie sich bei Röttgen und Stübgen findet. Auch dominieren Kontrollfunktionen, je stärker europäische Integration als Instrument der Mitgliedstaaten betrachtet wird.

Röttgen (2009b, 26257Df.) und Sarrazin (2009c, 26352Cf.) positionieren den Bundestag vor allem in inhaltlich-gestaltenden Funktionen, sowohl in der Primärrechtsetzung im Sinne eines Voranschreitens als auch in der Sekundärrechtsetzung, auf der der Fokus liegt. Vor dem Hintergrund des Legitimitätskonzepts, in dem die EU Zugriff auf übernationale Problembereiche schafft, erklärt sich das Abzielen auf die Nutzung des eröffneten Handlungspotentials. Röttgen (2009b, 26257D) begrüßt das Begleitgesetz mit der Begründung, dieses ermögliche „dem Parlament die politische Debatte [...] - und das nicht, nachdem die Würfel gefallen sind, sondern in der Gestaltung europäischer Politik hier im Parlament“.

Dies steht in Verbindung mit der Öffentlichkeit der parlamentarischen Debatte und einer erwarteten höheren Akzeptanz in der Bevölkerung (Norbert Röttgen 2009b, 26258A). Dem Verhältnis Bürger - Parlament kommt bei Sarrazin (2009c, 26352Cf.) zentrale Bedeutung zu, sowohl hinsichtlich der Kommunikation, der Repräsentation als auch der Verantwortung vor den Bürgern:

„[...] [W] ir als Abgeordnete des Deutschen Bundestages sind verantwortlich, treten ein, fühlen uns zuständig, informieren und diskutieren über das, was in Europa passiert. [...] Das erklären wir unseren Wählern. Es liegt an uns, was in Europa passiert. Wenn es an uns Bundestagsabgeordneten liegt, dann liegt es auch an unseren Wählerinnen und Wählern, dass Europa in der Integration weiter fortschreiten kann."

Zwar rekurriert auch Andreas Schockenhoff auf das Verhältnis zur Bevölkerung, allerdings indirekt und bezogen auf kontrollierende Tätigkeiten, die weder bei Sarrazin noch bei Röttgen von Belang sind. So solle der Bundestag dafür

„[... ] sorgen, dass die EU-Politik wieder bürgernäher wird. Dann müssen wir unsere Rechte aber auch nutzen und die im Begleitgesetz geschaffenen Kontrollmöglichkeiten anwenden: im Inneren gegenüber unserer eigenen Bundesregierung und nach außen, indem wir uns mit den anderen nationalen Parlamenten zügig koordinieren" (Andreas Schockenhoff 2008a, 15840Bf.).

Vor dem Hintergrund des Legitimitätsdenkens, das einer Delegationsbeziehung ähnelt, spiegeln sich die Aussagen der Prinzipal-Agent-Theorie, die die Kontrolle der Agenten durch ihre Prinzipale betont. Hier überwacht der Prinzipal Parlament den Agenten, die Regierung im Rat. Auch die angestrebte Koordinierung nationaler Parlamente deutet auf einen kontrollerleichternden Zusammenschluss der Prinzipale hin. ${ }^{55}$

Stübgen und Oppermann sehen für den Bundestag hinsichtlich Primärrechtsänderungen und gleichkommenden Maßnahmen vor allem eine Kontrollfunktion, denn er müsse „die Änderungen im europäischen Vertrag in jedem Einzelfall ausdrücklich erlauben“ (Thomas Oppermann 2009b, 26253D; vgl. Michael Stübgen 2009a, 25576B). Der Fokus auf kontrollierende Tätigkeiten setzt sich in der Sekundärrechtsetzung fort, insbesondere in der Kontrolle der Subsidiaritätseinhaltung. Entsprechend hatte Stübgen (2008b, 16474Cf.) eine

55 Vgl. Katrin Auel, Democratic Accountability and National Parliaments: Redefining the Impact of Parliamentary Scrutiny in EU Affairs, in: European Law Journal, 13. Jg. (2007), H. 4, S. 487 504, S. 496. 
nicht hinreichende Einhaltung durch europäische Institutionen bemängelt; zudem dient Subsidiarität der, im Legitimitätskonzept zentralen, Kompetenzeinhaltung. Ähnlich wie Stübgen findet Oppermann (2009b, 26253D), man könne das Verhältnis Bundestag - Bundesregierung ,auf die Formel bringen: uneingeschränktes Verhandlungsmandat nach außen, volle Rechenschaftspflicht nach innen“. Dabei betont er das Stellungnahmerecht des Bundestags, um gestalterischen Einfluss geltend zu machen (Thomas Oppermann 2009b, 26253D). Auf dieses ex ante Instrument gegenüber der Bundesregierung bezieht sich auch Link (2008b, 16466B), der möchte, „dass dieses Parlament sich mit den entsprechenden Fragen befasst und politisch mandatiert, bevor die entscheidenden Ministerratssitzungen stattfinden und im deutschen Namen abgestimmt wird“. Er setzt bei der legislativen Europäisierung an, die offenbar eine institutionelle Europäisierung ${ }^{56}$ (Michael Link 2008b, 16466B) evoziert, um gestalterischen Einfluss zu gewinnen. Auch richtet sich Link (2008b, 16466A), wie Toncar (2008a, 15846Df.), auf die Kontrolle der Subsidiaritätseinhaltung.

\section{2. (Ein)Blick von innen und Ausblick}

Die Standpunkte der MdBs zur Legitimität europäischen Regierens lassen sich vier Legitimitätsmustern zuordnen, die auf unterschiedlichen, teils inkompatiblen Begründungen von EU-Herrschaft basieren. Damit gehen auch andersgeartete Positionierungen hinsichtlich der vom Bundestag zu erfüllenden Funktionen einher. Insofern lässt die Mikroperspektive zumindest bezweifeln, dass eine Betrachtung des Bundestags lediglich als kollektiver Akteur ausreicht. Vielmehr treten binneninstitutionelle Konfliktlinien zutage, die sich weder eindeutig der Bruchlinie Regierungsmehrheit - Opposition noch Fraktionsgrenzen zuordnen lassen. ${ }^{57}$ Damit verbunden sind mehrere Erkenntnisse für die verknüpften Debatten: Erstens gelten offenbar für unterschiedliche Politikfelder andere (und verschieden konfliktive) Legitimationen mit divergierendem Legitimationsbedarf, wie sich insbesondere an den entgegengesetzten Positionen zur europäischen Sozialpolitik und zum Raum der Freiheit, der Sicherheit und des Rechts gezeigt hat. Eingehende Analysen einzelner Politikentscheidungen aus verschiedenen Politikfeldern (mit ungleichem Integrationsgrad und Entscheidungsverfahren) könnten hier Einsichten erbringen, inwieweit sich die im Legitimitätsdenken aufzufindenden evaluativ-kritischen Haltungen widerspiegeln.

Zweitens können diese binneninstitutionellen Friktionen in einer weiteren Schwächung des Bundestags gegenüber anderen europapolitischen Akteuren, insbesondere der Bundesregierung, resultieren. Gleichzeitig ist es aus der Perspektive der Regierung in diesem Gewirr von Stimmen nicht mehr eindeutig, welchem parlamentarischen Willen Gehör geschenkt und gefolgt werden soll.

Damit lassen die Ergebnisse, drittens, die begründete Vermutung zu, dass der Grad, bis zu welchem der Bundestag „Verlierer“ ${ }^{58}$ der Integration ist, nicht nur von strukturell ungünstigen Bedingungen, sondern zusätzlich von Konflikthaftigkeit und (In-)Kompatibilität

56 Vgl. Annette Elisabeth Töller, a.a.O. (Fn. 8).

57 Vgl. auch Andreas Wimmel, Neue (alte) Konfliktlinien in der Europapolitik: Die Parlamentsdebatte zum Vertrag von Lissabon im Deutschen Bundestag, in: ZParl, 40. Jg. (2009), H. 4, S. 746 758.

58 Andreas Maurer / Wolfgang Wessels (Hrsg.), a.a.O. (Fn. 7). 
interner Vorstellungen abhängt. Gerade angesichts der Konfliktlinien, die quer zu den klassischen (Soll-)Bruchstellen laufen, wäre eine vertiefende Betrachtung der innerparlamentarischen Aushandlungsprozesse und Mechanismen, die zur Durchsetzung einer bestimmten Position führen, aufschlussreich. So wirft die Vielschichtigkeit sowohl der Legitimitätsmuster als auch, und damit korrespondierend, der Positionierung des Bundestags die Frage auf, wie diese in kollektives Handeln des Bundestags übersetzt werden: Welche Position setzt sich wann und wie durch? Ob und unter welchen Bedingungen hemmen sich konfligierende Deutungen gegenseitig und tragen zu der beklagten Inaktivität des Bundestags bei?

Insgesamt hat, viertens, die Verlagerung auf die Mikroebene und die Verwendung diskursanalytischer Werkzeuge eröffnet, dass ein plausibler Zusammenhang zwischen der Positionierung des Bundestags und Legitimitätsmustern besteht. Durch die in die Tiefe gehende Betrachtung wurde deutlich, dass es nicht ausreicht, eine Analyse des Legitimitätsdenkens auf idealtypische Finalitätsvorstellungen oder reduzierte Narrative zu begrenzen. Naturgemäß konnte lediglich ein Ausschnitt der Realität (sowohl der Akteure als auch in zeitlicher Perspektive) betrachtet werden, so dass die gezogenen Schlüsse tentativ bleiben. Eine Ausweitung des zeitlichen Horizonts und die Erhebung und Analyse vergleichbarer Daten in diesem Rahmen ist daher anzuregen. Vielversprechend scheinen hier die zunehmenden Bemühungen der Critical Discourse Analysis zu sein, verstärkt dem Dreieck Diskurs-Kognition-Gesellschaft/Umwelt und dessen Dynamik Rechnung zu tragen ${ }^{59}$, womit sich eine Verknüpfung linguistischer und politikwissenschaftlicher Forschung anbietet.

59 Vgl. Teun van Dijk, Critical Discourse Studies: A Sociocognitive Approach, in: Ruth Wodak I Michael Meyer (Hrsg.), Methods of Critical Discourse Analysis, London 2009, S. 62 - 86; Ruth Wodak, ,Doing Europe': The Discursive Construction of European Identities, in: Richard Mole (Hrsg.), Discursive Constructions of Identity in European Politics, Houndmills 2007, S. 70 - 94.

\title{
Subsidiarität und Mitsprache. Nationale Parlamente nach Lissabon
}

\author{
Aron Buzogány und Andrej Stuchlik
}

Innerhalb kurzer Zeit haben die formalen Instrumente der Subsidiaritätskontrolle, insbesondere der parlamentarische Frühwarnmechanismus des Lissabon-Vertrages ${ }^{1}$, bei Praktikern wie in der Wissenschaft viel Beachtung erfahren. Grund dieser Aufmerksamkeit ist die damit verbundene Aussicht, mittels neuer ex-ante Kontrollverfahren die Rolle der nationa-

1 Vertrag von Lissabon zur Änderung des Vertrags über die Europäische Union und des Vertrags zur Gründung der Europäischen Gemeinschaft vom 13. Dezember 2007, ABl. C306 vom 17. Dezember 2007 (nachfolgend Lissabon-Vertrag), in Kraft getreten am 1. Dezember 2009. Siehe auch Protokoll über die Rolle der nationalen Parlamente in der Europäischen Union, ABl. C 306/148 (nachfolgend Parlamente-Protokoll); Protokoll über die Anwendung der Grundsätze der Subsidiarität und der Verhältnismäßigkeit, ABl. C306/150 (nachfolgend Subsidiaritäts-Protokoll). 